\title{
A macroscale finite element approach for simulating the bending behaviour of
}

\section{biaxial fabrics}

F. Yua, S. Chen ${ }^{a}$, J.V. Viisainen ${ }^{b}$, M.P.F. Sutcliffe ${ }^{b}$, L.T. Harper, ${ }^{a}$, N.A. Warrior ${ }^{a}$

a Composites Research Group, University of Nottingham, Nottingham, UK

${ }^{\mathrm{b}}$ Department of Engineering, University of Cambridge, Cambridge, UK

Corresponding author: L. T. Harper

E-mail address: (lee.harper@nottingham.ac.uk)

\begin{abstract}
A macroscale finite element (FE) model was developed to simulate the forming behaviour of biaxial fabrics, incorporating the effects of bending stiffness to predict fabric wrinkling. The dependency of the bending stiffness on the fibre orientation was addressed by extending a non-orthogonal constitutive framework previously developed for biaxial fabric materials. The nonlinear bending behaviour of a biaxial non-crimp fabric (NCF) with pillar stitches was characterised by a revised cantilever test using structured light scanning to measure specimen curvature, providing input data for the material model. Simulations were performed to replicate the bias-extension behaviour of the NCF material, showing good agreement with experimental data. Wrinkles were observed within the central area of the specimen at low extension, which consequently affect the uniformity of the shear angle distribution in the region where pure shear is expected.
\end{abstract}

\section{Keywords}

A. Fabrics/Textiles; A. Preform; C. Finite element analysis (FEA); E. Mechanical property. 


\section{Introduction}

Volume production of composite structures incorporating biaxial fabrics requires a preforming step to convert flat 2D plies into complex 3D shapes prior to liquid moulding. Due to large deformations during forming, significant manufacturing defects in the form of fabric wrinkles can be generated. A number of studies have been devoted to shear induced wrinkling for woven fabrics [1-4], concluding that local fabric thickening caused by yarn compaction is the key factor in wrinkle formation. Consequently, criteria based on the "shear locking angle" is typically used to determine possible wrinkle locations from the shear angle distribution predicted by kinematic models [5] or FE simulations using membrane elements [6]. Whilst these tools provide designers with varying degrees of confidence for optimising the forming process $[7,8]$, the severity of wrinkling in problem areas is largely unknown since the bending stiffness of the fabric is overlooked.

Recent studies have reported that the magnitude of the shear angle is not directly related to the severity of fabric wrinkling, and that the bending stiffness of fabrics is of greater importance to the shape and number of wrinkles [9]. Especially, in diaphragm forming where fabrics are typically constrained much less than in press forming, out-of-plane wrinkling is the most common form of manufacturing defect [7]. Interactions between individual plies was shown to play an important role in the formation of wrinkles when forming a stack of plies over a double curvature tool [10]. Also, wrinkle formation is related to the fabric architecture, in particular the stitch pattern in non-crimp fabrics (NCFs) [11, 12].

Wrinkles are often observed during material characterisation tests, such as the bias extension or picture frame shear tests [13-15] for determining the in-plane shear resistance of fabrics. Whilst this deformation is useful for determining the "shear locking angle" to quantify the severity of yarn compaction, the onset of wrinkling can produce inaccuracies in the measured shear angle during the test $[16,17]$. Shear induced wrinkling can be delayed or prevented in picture frame shear testing due to small tensile forces generated along the fibres by the clamps [18], whereas bias extension testing 
is quite sensitive to shear induced fabric buckling as the vertical specimen edges are unconstrained. To reduce the error caused by fabric waviness, a 3D stereoscopic Digital Image Correlation (DIC) system was suggested in [16] to produce more accurate measurements for in-plane fibre angles following wrinkling. However, it is understood that wrinkling will still produce an out-of-plane perturbation to the in-plane shear kinematics of the specimen.

The objective of the current work is to develop a robust simulation tool to predict fabric wrinkling during forming. A Composite Shell Element available in Abaqus/Explicit is implemented within an existing macroscale material model [8] to capture the effects of fabric bending. The performance of the proposed method is evaluated by comparing predictions with reported experimental data for both a woven fabric [19] and a NCF [6]. A Structured White Light Scanner (SWLS) has been employed to measure the curvature of NCF specimens during a cantilevered bend test, in order to determine the bending stiffness along the fibre directions. The resulting bending behaviour is incorporated into the proposed simulation framework to investigate the influence of the pillar stitch on wrinkle development. The model is validated by comparing predicted wrinkle amplitudes with experimental data taken from bias extension tests.

\section{Fabric characterisation}

\subsection{Material}

A biaxial carbon fibre NCF (Hexcel FCIM359) was used in this study. The fabric consists of two unidirectional plies, with yarn orientations at $\pm 45^{\circ}$, assembled by pillar stitches in the $0^{\circ}$ direction. The material parameters are listed in Table 1. The shear resistance for this fabric was determined using the picture frame shear test in previous work by the authors [6], as shown in Figure 1. The bending behaviour was characterised by a modified cantilever test in the current work, as described in Section 2.2 . 


\subsection{Bending stiffness}

Cantilever tests have been widely used to characterise fabric specimens with either linear $[20,21]$ or non-linear [22, 23] bending behaviours. According to BS EN ISO 9073-7 [21], a cantilevered fabric strip subjected to gravity, exhibits a constant bending stiffness per unit length, $B$, for a given cantilever overhang, $L$, and areal weight, $w$ :

$$
B=\frac{1}{\frac{\tan (\phi)}{\cos (0.5 \phi)}} \times \frac{w L^{3}}{8}
$$

The angular deflection, $\phi$, is the angle measured from the horizontal to the secant line, drawn between the fixed end and the free end of the cantilevered fabric strip. Typically, either $\phi=41.5^{\circ}$ or $\phi$ $=7 \cdot 1^{\circ}$ is recommended for the measurement [21].

The NCF material was initially tested according to the standard cantilever test outlined in BS EN ISO 9073-7 [21]. Fabric specimens were cut by a rotary knife into $250 \mathrm{~mm} \times 30 \mathrm{~mm}$ strips, containing 20 fibre bundles across the width of each specimen. A steel rule was used to set the overhang length of each fabric specimen on the rig, as shown in Figure 2. A rubber layer was added to the surface of the rule to increase the coefficient of friction, preventing relative movement between the rule and the fabric specimen. Each specimen was pushed forward over the clamping edge until the tip of the fabric specimen touched the slope at the desired angle $\phi$. Six specimens were tested in two different configurations as the bending stiffness may depend on the fibre architecture in the through-thickness direction (see Figure 3 ). The $0^{\circ}$ fibres were positioned on both the uppermost surface and the lowermost surface about the mid-plane of the specimen.

Results for both bending configurations are compared in Figure 3, indicating that the macroscale bending response is dependent on the bending direction. For an angular deflection value of $7.1^{\circ}$, the stiffness in the positive bending direction is $0.0073 \mathrm{~N} \cdot \mathrm{m}$ compared to $0.0057 \mathrm{~N} \cdot \mathrm{m}$ for the negative direction. In addition, the bending stiffness calculated from the large deflection angle $\left(41.5^{\circ}\right)$ is lower 
than the value resulting from the small deflection angle $\left(7.1^{\circ}\right)$, which confirms that the bending stiffness for this NCF fabric is non-linear.

In order to characterise the non-linear bending behaviour of this material, a revised cantilever bending testing method was used [23]. Bending moments and curvatures were not determined as a function of loading. A curve was drawn using a single loading configuration, establishing the curvature and the bending moment as functions of curvilinear coordinates along the specimen. A Structured White Light Scanner (HP-Pro S3) (SWLS) was employed to provide an accurate representation of the curvature of the specimen, avoiding parallax error commonly experienced with other photogrammetry methods. The overhang length was fixed to be $210 \mathrm{~mm}$ to ensure a wide range of curvature along the specimen. 3D point clouds of the deformed specimens were acquired, with a precision of up to $0.05 \%$ over the scan area $(210 \mathrm{~mm} \times 30 \mathrm{~mm})$. Due to potential twist of the specimen, measurements were taken over the centre of the specimen corresponding to $5 \%$ of the specimen width (the yellow strip in Figure $4 a$ ). A polynomial was used to fit the deflection curve, but it is possible for negative curvatures to occur at certain points due to fitting errors. The order of the fitting function was therefore adjusted to ensure a positive and monotonic curvature variation for a wide range of the deflection curve. Consequently, problem areas were restricted to either the free tip or the fixed end of the cantilevered specimen. For the NCF studied, the deflection curve was obtained by fitting a $6^{\text {th }}$ order polynomial function to the projected points from six specimens. As an alternative method, the bending moment-curvature relation can be determined from the relation between cantilever overhangs and deflection angles measured by a bespoke testing rig [24], which avoids measuring the entire scale of the cantilevered specimen.

The bending moment $M$ and the curvature $\kappa$ at an arbitrary point $X(x, y(x))$ on the deflection curve (See Figure 4) were calculated as 


$$
\begin{gathered}
M=\int_{S}^{L} \overrightarrow{X P} \times \vec{w} \mathrm{~d} u \\
\kappa=\frac{y^{\prime \prime}}{\left(1+y^{\prime 2}\right)^{3 / 2}}
\end{gathered}
$$

where $s$ is the curvilinear abscissa of Point $X, L$ is the length of overhang, $\vec{w}$ is the vector of the weight per unit length of the fabric specimen; $u$ is the Frenet's coordinate of Point $P$ moving along the segment between Point $X$ and the tip of overhang E. $y^{\prime}$ and $y^{\prime \prime}$ in Eq.(3) are the first and second derivatives of the fitted deflection curve with respect to $x$, see Figure 4.

The relationship between $M$ and $\kappa$ was fitted using Voce's model [25], where the root mean square error (RMSE) was calculated to be less than $5 \%$ of the experimental data:

$$
M(\kappa)=R_{0} \cdot \kappa+R_{\infty}\left[1-\exp \left(-\kappa / \kappa_{\lim }\right)\right]
$$

Here, $R_{0}$ and $R_{\infty}$ are fitting constants and $\kappa_{\lim }$ is the exponential saturation parameter.

As shown in Figure 5, the obtained $M(\kappa)$ curves for the two bending directions are similar, but exhibit different gradients at the start and end of the curves. This indicates the dependency of the bending stiffness on the mesoscale architecture, which may be more pronounced for different NCF stitch patterns. The slopes of the curves at zero curvature are $0.008 \mathrm{~N} \cdot \mathrm{m}$ and $0.0056 \mathrm{~N} \cdot \mathrm{m}$ for the positive and negative bending directions respectively, which are close to the bending rigidities measured by the standard testing method at $\phi=7.1^{\circ}$. This also indicates that the bending behaviour is approximately linear elastic under small curvatures. By scanning the surface of the bent specimen (see Figure 6) using the SWLS, local buckling of the unidirectional layer can be observed on the compressive side of the specimen for each fibre orientation, which compromises the bending resistance of the fabric under large curvature. It is also worth noting that the bending stiffness (i.e. the slope of the $M$ ( $\kappa)$ curve) decreases with the increase of curvature under both positive and negative bending.

\section{Material modelling}




\subsection{Background}

Computationally efficient modelling techniques are required to account for fabric bending effects during forming, in order to investigate the influence of out-of-plane wrinkles. Mesoscale modelling of interlaced yarns and their interactions $[26,27]$ is impractical for simulating the forming behaviour of industrial-scale components, due to the computational resources required. Moreover, detailed mechanical input properties for the fabric constituents at the meso-scale are generally too difficult to characterise by standard testing methods. At the macroscale, the fabric is homogenised and considered to be a continuum to offer savings in run time. A hyper-elastic constitutive law [28] was previously implemented using continuum elements to describe large deformations for thick inter-lock textile preforms, whereas non-orthogonal hypo-elastic approaches $[8,29,30]$ are typically used for thin fabric sheets undergoing large shear deformation. Due to the relative movement of fibres, the flexural rigidity of fabrics determined from the tensile stiffness of yarns based on classical beam/shell theory is typically higher than experimental values [31]. To account for this in numerical simulations, different values for tensile and compressive moduli were assigned to the integration points through the thickness of the shell [30] or beam [32] elements in a hybrid mesh. This method is intuitive, but is unable to independently control bending and membrane rigidities, and may lead to unrealistic results when the element cross-section is subjected to combined loads. A more sophisticated pantographic mesh has been developed by replicating representative fabric unit cells using a combination of structural elements $[19,33]$, with the aim of comprehensively incorporating forming mechanisms into the FE model. However, the reported computational cost of this method is still high due to the large number of degrees of freedom (DOFs) introduced by structural elements and connectors. Semidiscrete shell elements [34] were formulated to decouple the membrane and bending behaviours of fabrics, but these are not readily available in commercial FE codes. A rate-dependent viscoelastic bending model for fabrics was developed using an Abaqus user-defined general shell section UGENS [35], where the membrane and bending contributions were captured separately by membrane and shell elements sharing common nodes. Typically, a constant bending stiffness is used for simplicity, 
but non-linear bending behaviour has been observed in experimental studies [23] due to the friction between fibres and local fibre buckling, as shown in Figure 6. Also, different bending stiffness was reported for biaxial NCFs made up of two stitched unidirectional plies when tested at different specimen orientations [36].

A novel method is proposed to account for the bending behaviour of fabrics during forming, using a laminated shell approach to decouple the out-of-plane bending behaviour from the in-plane tensile and shear behaviours. This is efficiently implemented using a laminated shell element from the Composite Layup Toolset in Abaqus/Explicit. Each fabric ply is represented by a single layer of shell elements, leading to a reduction in the number of degrees of freedom compared to previously used hybrid meshes [19, 32, 33, 35].

\subsection{Decoupling membrane and bending stiffness}

The bending stiffness of fabrics is not directly related to the tensile modulus of fibre material due to fibre slippage and tow buckling. This renders classic beam or shell elements invalid for modelling the bending behaviour of these fabric materials. According to Döbrich et al. [36], the membrane and bending behaviours of NCFs can be decoupled, therefore in the current work the Abaqus Composites Layup Toolset was employed to assemble these individually defined contributions using a laminated shell element. As shown in Figure 7, decoupling the membrane and bending stiffness was achieved by tailoring the layer thickness and the number of integration points within the layup. Three artificial layers were defined for each shell element (S4R), where the two outer surface layers were used to define the bending behaviour, while the central layer was used to control the membrane behaviour. The total thickness of the element, $h$, was the same as the fabric thickness, and the thickness of the two surface layers were assumed to be identical. The bending moment along each fibre direction in the deformed configuration, $M_{f i}$, acting at the shell cross-section, was calculated by integrating the moment resulting from the fibre stress $\sigma_{f i}(z)$ at the integration points over the thickness of the shell element. 


$$
M_{f i}=\int_{-h / 2}^{+h / 2}\left\{\sigma_{f i}(z) \cdot \bar{F}_{33}^{2} \cdot z\right\} d z .
$$

Due to the incompressibility of the shell element, the thickness change $\bar{F}_{33}$ was determined by the inplane components of the deformation gradient $\bar{F}_{i j}(i, j=1,2)$, i.e.

$$
\bar{F}_{33}=\frac{1}{\bar{F}_{11} \bar{F}_{22}-\bar{F}_{12} \bar{F}_{21}} \text {. }
$$

Equation (5) implies that the stresses on the shell reference surface (i.e. the mid-plane of the shell element, where $z=0$ ) has no contribution to the out-of-plane bending moment (or stiffness) of the shell element. Thus, a single integration point was assigned to the central layer to eliminate its bending contribution to the laminate layup. As a result, the bending stiffness only depends on the properties of the two surface layers.

Since the thickness of each fabric ply is much smaller than the in-plane dimensions of the specimen blank, the ply can be assumed to be a thin-walled shell in order to derive the macro bending resistance per unit length in the fibre directions. An identical Young's modulus (i.e. $E_{f i}^{\operatorname{surf}}$ ) was assigned to the top and bottom surface layers, whereby the bending stiffness in the $i^{\text {th }}$ fibre direction (i.e. $B_{f i}, i=1,2$ ) was obtained as

$$
B_{f i}=\frac{1}{12} E_{f i}^{\operatorname{surf}}\left[h^{3}-\left(t^{\text {ctral }}\right)^{3}\right]
$$

where, $t^{\text {ctral }}$ denotes the thickness of the central layer.

According to Eq. (7), the expected bending stiffness $B_{f i}$ can be achieved by adjusting either $E_{f i}^{s u r f}$ or $t^{c t r a l}$ or both. However, modifying $E_{f i}^{\operatorname{surf}}$ is considered to be the most practical way to control the bending stiffness $B_{f i}$ due to a linear relationship between them.

Since the stress-strain relationship at the integration points was assumed to be elastic, a three-point Simpson's integration approach is sufficient for calculating the bending moment on the shell crosssection. Only one integration point was therefore assigned to each surface layer to reduce the 
computational cost. Thus, three integration points in total were used through the thickness direction of each fabric ply, eliminating the inertial moment of each layer with respect to its centroid axis. Therefore, the bending stiffness parallel to the fibres, $B_{f i}^{\prime}$, can be written as

$$
B_{f i}^{\prime}=\frac{1}{16} E_{f i}^{\operatorname{surf}}\left(h-t^{\text {ctral }}\right)\left(h+t^{\text {ctral }}\right)^{2}
$$

The nominal Young's modulus of each fabric ply along the ith yarn (i.e. $E_{f i}^{l a m}$ ) was determined according to the Rule of Mixtures

$$
E_{f i}^{\operatorname{lam}}=E_{f i}^{\operatorname{surf}}\left(\frac{h-t^{c t r a l}}{h}\right)+E_{c t r a l}\left(\frac{t^{c t r a l}}{h}\right)
$$

In practice, the laminate modulus, $E_{f i}^{\operatorname{lam}}$, and the bending stiffness, $B_{f i}$ or $B_{f i}^{\prime}$, are input parameters, based on which the moduli values of each layer within the layup were determined. These were updated during the analysis to approximate the measured tensile and bending stiffness for each fibre direction.

\subsection{Relating bending stiffness to yarn directions}

As shown in Figure 7, an established non-orthogonal constitutive model [6] was modified to define the bending contribution of each primary yarn based on the current fibre orientation frame, which is dependent on the rotation of the yarns during in-plane shear. The bending property of each yarn was added to the constitutive model by updating the moduli values of three layers within the composite shell element for each fibre direction. This approach enables the change in fibre orientation to be tracked during forming, updating the bending stiffness term as the fabric shears in-plane. The material model was implemented through a user material subroutine (VUMAT) based on Abaqus/Explicit. Detailed numerical implementation of the non-orthogonal material model is available in the literature for a woven fabric [29] and a non-crimp fabric [8]. Unlike the hybrid beam-membrane/shell meshes reported in the literature [32, 33], where bending stiffness is considered via additional elements with 
rotational degrees of freedom (DOFs), the current method requires less DOFs and provides meshindependency for non-orthogonal fibres.

\subsection{Curvature updating}

According to the Koiter-Sanders shell theory [37], in-plane strain at an integration point through the thickness of the S4R element can be determined by superposition of the membrane strain on the reference surface, $\bar{\varepsilon}$ and the bending strain related to the element curvature, $\kappa$. Thus, the fibre strain, $\varepsilon_{f i},(i=1,2)$, in the fibre coordinate system can be determined by the fibre strain on the shell reference surface (i.e. $\bar{\varepsilon}_{f i}$ ) and the curvature of reference surface (i.e. $\kappa_{f i}$ ):

$$
\varepsilon_{f i}=\bar{\varepsilon}_{f i}+\bar{F}_{33} Z_{0} \kappa_{f i}
$$

where, $z_{0}$ is the initial distance from the integration point to the mid-plane of the shell element and $\bar{F}_{33}$ is the thickness change defined in Eq. (6).

The procedure for updating the bending moment and curvature is shown in Figure 8. During each time increment, the non-orthogonal constitutive framework is employed to determine the strain along each fibre direction. Eq. (10) is then used to calculate the current curvature of each yarn, which relates to the bending stiffness. Thus, their relationship should be measured as material inputs to update the bending stiffness. This procedure was implemented in a VUMAT based on Abaqus/Explicit. However, the calculation of the bending moment and curvature in the fibre parallel systems for each element requires data from adjacent integration points in the thickness direction, which is not directly available in the VUMAT. Therefore, a user defined subroutine VEXTERNALDB was employed to access an external database for passing updated element information between time increments.

\section{Model validation}




\subsection{In-plane shear}

Both picture frame and bias-extension tests were simulated to confirm that decoupling the membrane stiffness and the bending stiffness does not have an adverse effect on the in-plane shear response. Shear compliance curves for a woven fabric [19] and an NCF [6] were taken from the literature (see Table 2). In order to reduce the computational time, the axial Young's modulus along the fibre direction was chosen to be $3 \mathrm{GPa}$ throughout this work and the fibre strain was limited to $<1 \%$, as recommended by Harrison et al [19]. All fabric plies were modelled using Abaqus shell elements (S4R) with an edge size of $3 \mathrm{~mm} \times 3 \mathrm{~mm}$, as established by a mesh density study in [6]. As shown in Figure 9, the curve for the normalised shear force (cross-head force normalised by the edge length of the shear region) predicted by the current model for the woven fabric closely mimics the curve predicted by the discrete model presented by Harrison et al [19]. The simulated wrinkle onset angle $\left(\sim 41^{\circ}\right)$ is slightly higher than the experimentally obtained value $\left(3^{\circ}\right)$ in Harrison's paper [19]. For the FCIM359 NCF [6], the predicted cross-head force closely matches the experiment data in Figure 1, with the RMSE less than $3 \%$ of the peak value taken from the mean experimental curve. These comparisons indicate that the predicted in-plane shear behaviour is not adversely affected by incorporating the bending stiffness term into the model for both woven fabrics and NCF.

\subsection{Bending}

The revised cantilever test for NCF coupons was simulated for both positive and negative bending configurations, by implementing the non-linear bending behaviour characterised in Section 2.2. These simulations are compared against experimental data in Figure 10. The edge profile of the simulated coupons falls within the corresponding error bars from the experimental study. The difference in the tip deflection between the experimental coupons and the profile predicted by the non-linear bending model is $<0.5 \mathrm{~mm}$ for positive bending and $<1.5 \mathrm{~mm}$ for negative bending. A small difference in angular deflections (less than $4^{\circ}$ ) is observed between the positive and negative bending directions. For comparison, simulations were also performed using a constant bending stiffness value measured by 
the standard cantilever test at an angular deflection of $41.5^{\circ}$ (denoted as FEA, $\mathrm{B}_{41.5}$ in Figure 10). The deflected shape sits significantly above the experimental data, indicating that the standard cantilever test [21] may not be suitable for these engineered fabrics.

\subsection{Out-of-plane wrinkling}

Bias extension testing was used to generate controlled macroscale out-of-plane wrinkles in fabric samples. The non-linear $M(\kappa)$ bending curve from Section 2.2 and the in-plane shear curve produced by Chen et al [6] were used as material model inputs to simulate the bias-extension test for the NCF material (FCIM359). For the bias-extension simulation, one short edge of the rectangular specimen was fixed, while a $40 \mathrm{~mm}$ displacement was applied to the opposite edge. The NCF was modelled using quadrilateral laminate shell elements $(2 \mathrm{~mm} \times 2 \mathrm{~mm}$ ) with reduced integration (S4R). The true areal density of the NCF $\left(0.44 \mathrm{~kg} / \mathrm{m}^{2}\right)$ was assigned to the FE model. The run time per simulation was 5.6 hours (computer specification: Intel Xeon CPU E5-1620 v3 @ 3.50 GHz; 64.0 GB, 64-bit) with a step time of 5 seconds.

The surface waviness of wrinkled specimens was obtained by both Digital Image Correlation and SWLS. All tests were carried out on a universal testing machine at a crosshead speed of $20 \mathrm{~mm} / \mathrm{min}$. A GOM DIC setup with two cameras was mounted in front of the testing machine to monitor the deformation of the speckled specimen, enabling full-field out-of-plane displacements and local shear angles to be calculated. Shear angle variations at individual points were obtained by correlating the relative angle from facet points (subset size: $15 \times 15$ pixels) located along the fibre tows. As suggested in [19], the initiation point of wrinkling in the bias-extension test was captured when the wrinkle amplitude at the central cross-section of the specimen first exceeded $1 \mathrm{~mm}$. Open-source software, CloudCompare [38], was used to determine wrinkle amplitudes at different cross-head displacements by comparing the point cloud of the deformed specimen against the reference cloud at zero extension. 
Out-of-plane displacements from the FE simulations are compared to experimental values for different loading stages in Figure 11. Fabric wrinkling starts when the shear deformation reaches an angle of $8^{\circ}$ (extension $=7.5 \mathrm{~mm}$ ) due to yarn compaction and the absence of constraints along the free edges of the specimen. However, this shear angle may not be a suitable threshold for the onset of wrinkling during forming, since tension in the fibres and through-thickness compression from the blank holder may prevent wrinkles from occurring. The wrinkle amplitude rapidly increases to a peak value $(\sim 3.6 \mathrm{~mm})$ at an extension of $14.1 \mathrm{~mm}$. The maximum out-of-plane displacement varies within the range of 3.0-3.5 mm until stitches start to fail at an extension of $23.0 \mathrm{~mm}$. The wrinkle patterns monitored by DIC and SWLS are approximately symmetric about the central axis in the loading direction, with the greatest waviness occurring in the centre of the specimen (see Section A-A in Figure 11), which agrees with the FE simulations. The amplitude of the largest wrinkle taken from the simulation is compared against DIC and SWLS measurements for 12 specimen repeats in Figure 12. There is a good agreement between the experimental and simulation data sets (RMSE $=0.09 \mathrm{~mm}$, which is $2.5 \%$ of the maximum amplitude). The DIC setup was unable to track the speckle pattern on the specimen once stitches started to fail and intra-ply fibre slippage occurred. In comparison, SWLS was more effective for characterising out-of-plane wrinkles throughout the test. According to the SWLS data in Figure 11, the waviness amplitude and the wrinkled area both decrease due to the release of constraints, implying that the local removal of intra-ply stitches could be a potential way to eliminate forming-induced wrinkles for biaxial NCFs.

\section{Validity of the bias-extension test}

Whilst the bias-extension test is simple to perform, it is difficult to ensure uniform shear within the central diamond region of the specimen (the region indicated by the black dashed line in Figure 13) when fabric wrinkling occurs or stitches fail. This can clearly affect the measured shear angle distribution, causing inaccurate shear compliance curves [16]. As shown in Figure 13a, the shear angle distribution in the central diamond region of a bias extension specimen becomes non-uniform as a result of wrinkling, even though the shear deformation is small. The pure shear condition in the bias- 
extension test is disrupted by local stitch failure at an extension of $30 \mathrm{~mm}$. In comparison, as shown in Figure 13b, the picture frame shear test at the same shear angle is able to ensure uniform shearing due to the absence of wrinkling in the central zone. The pure shear condition is maintained across the entire specimen area within the 4-bar linkage system, although stitches have failed. Therefore, the picture frame shear test is considered to be a more relevant test for characterising the in-plane shear behaviour of biaxial NCF fabrics.

The severity of the non-uniform shear condition in bias extension testing has been explored further by considering the shear angle distribution at two discrete points within the central diamond shaped "pure shear" zone, marked A and B (see Figure 13a). Average shear angles derived from DIC measurements taken from the surface of six specimen repeats are plotted in Figure 14 for points $\mathrm{A}$ and B. For comparison, shear angles from the FEA simulations are also included. All curves appear to be similar prior to wrinkling, but all are lower than the theoretical curve [19] which assumes a perfect pin-jointed net. This discrepancy has previously been attributed to intra-ply fibre slippage and size effects caused by the specimen width [39].

The shear angles at Points A and B increase linearly with the crosshead displacement, but then deviate from each other after wrinkling occurs at an extension of $7.5 \mathrm{~mm}$. This confirms that the shear angle distribution becomes non-uniform when wrinkling starts, and therefore the pure shear assumption in the central diamond region is no longer valid. The difference in shear angle at Points A and B increases gradually, reaching a maximum difference of $8^{\circ}$ before the DIC speckle pattern breaks down (extension of $20 \mathrm{~mm}$ ). The bifurcation point at an extension of $7.5 \mathrm{~mm}$ denotes the onset of wrinkling in this bias extension test, raising concerns over the quality of the in-plane shear data. Although the measured shear angle variation at Point $A$ is close to the theoretical curve, it does not match the shear load derived from the crosshead force based on the assumption of an ideal pin-jointed net. This is due to the redistribution of shear load within the central diamond region. 


\section{Conclusions}

A macro-scale FE model was developed to simulate fabric forming behaviour. Fibre-orientation dependent non-linear bending was integrated into a non-orthogonal fabric model. The mechanical behaviour of the fabric was defined using a material subroutine in Abaqus/Explicit, avoiding the need for a bespoke element formulation. Comparisons with experimental data have shown the suitability of the proposed method to replicate the macroscale deformation of bi-axial fabrics.

Bending behaviour of a pillar-stitched NCF was characterised for both positive and negative bending configurations by a revised cantilever test. A polynomial function was used to fit the deflection shape acquired by a Structured White Light Scanner (SWLS) to obtain the non-linear bending moment as a function of curvature at all points along the specimen. The model was validated by studying out-ofplane wrinkle formation during bias extension testing. A combination of digital image correlation (DIC) and SWLS were used to measure the wrinkle amplitude from experimental bias extension coupons. The predicted shape and magnitude of wrinkles matched well with the experimental data, with the root mean square error of the largest wrinkle amplitude being less than $3 \%$. Wrinkles were initiated at a small shear deformation and the amplitude of these wrinkles remained constant until stitches started to fail. Subsequently, the wrinkle amplitude reduced as stitches progressively failed, indicating that the in-plane shear compliance affects the onset and propagation of wrinkles. Reducing the fabric shear resistance by locally removing stitches may therefore be a potential way to reduce or delay the onset of wrinkling.

From both DIC measurements and the FEA simulation, it was concluded that the picture frame test is a more robust method for producing in-plane shear curves for NCF fabrics compared to the bias extension test. The bias extension test may not be able to produce uniform shearing in the central area of the specimen, due to the presence of out-of-plane wrinkles and stitch failure causing fibre slippage. 


\section{Acknowledgement}

This work was partially funded by the Engineering and Physical Sciences Research Council, as part of the "EPSRC Future Composites Manufacturing Research Hub" (EP/P006701/1), and via an "EPSRC Doctoral Training Partnership" studentship. The authors would also like to acknowledge funding from the China Scholarship Council (CSC) and the Aviation Industry Corporation of China (AVIC).

\section{REFERENCES}

[1] Prodromou, A.G. and J. Chen, On the relationship between shear angle and wrinkling of textile composite preforms, Composites Part A: Applied Science and Manufacturing, 28(5), 1997, pp. 491-503).

[2] Zhu, B., T. Yu, and X. Tao, An experimental study of in-plane large shear deformation of woven fabric composite, Composites Science and Technology, 67(2), 2007, pp. 252-261 (DOI: 10.1016/j.compscitech.2006.08.011).

[3] Long, A.C., C.D. Rudd, M. Blagdon, and P. Smith, Characterizing the processing and performance of aligned reinforcements during preform manufacture, Composites Part A: Applied Science and Manufacturing, 27(4), 1996, pp. 247-253 (DOI: https://doi.org/10.1016/1359-835X(95)00051-3).

[4] Mohammed, U., C. Lekakou, L. Dong, and M.G. Bader, Shear deformation and micromechanics of woven fabrics, Composites Part A: Applied Science and Manufacturing, 31(4), 2000, pp. 299-308 (DOI: https://doi.org/10.1016/S1359-835X(99)00081-0).

[5] Long, A.C., Composites forming technologies. 2007, Cambridge: Woodhead.

[6] Chen, S., O.P.L. McGregor, L.T. Harper, A. Endruweit, and N.A. Warrior, Defect formation during preforming of a bi-axial non-crimp fabric with a pillar stitch pattern, Composites Part A: Applied Science and Manufacturing, 91, 2016, pp. 156-167 (DOI: 10.1016/j.compositesa.2016.09.016).

[7] Chen, S., O.P.L. McGregor, A. Endruweit, M.T. Elsmore, D.S.A. De Focatiis, L.T. Harper, and N.A. Warrior, Double diaphragm forming simulation for complex composite structures, Composites Part A: Applied Science and Manufacturing, 95, 2017, pp. 346-358 (DOI: 10.1016/j.compositesa.2017.01.017).

[8] Chen, S., A. Endruweit, L.T. Harper, and N.A. Warrior, Inter-ply stitching optimisation of highly drapeable multi-ply preforms, Composites Part A: Applied Science and Manufacturing, 71, 2015, pp. 144-156 (DOI: 10.1016/j.compositesa.2015.01.016).

[9] Boisse, P., N. Hamila, E. Vidal-Sallé, and F. Dumont, Simulation of wrinkling during textile composite reinforcement forming. Influence of tensile, in-plane shear and bending stiffnesses, Composites Science and Technology, 71(5), 2011, pp. 683-692 (DOI: 10.1016/j.compscitech.2011.01.011).

[10] Guzman-Maldonado, E., P. Wang, N. Hamila, and P. Boisse, Experimental and numerical analysis of wrinkling during forming of multi-layered textile composites, Composite Structures, 208, 2019, pp. 213-223 (DOI: 10.1016/j.compstruct.2018.10.018).

[11] Krieger, H., T. Gries, and S.E. Stapleton, Shear and drape behavior of non-crimp fabrics based on stitching geometry, International Journal of Material Forming, 2017, pp. (DOI: 10.1007/s12289-017-1368-1).

[12] Arnold, S.E., M.P.F. Sutcliffe, and W.L.A. Oram, Experimental measurement of wrinkle formation during draping of non-crimp fabric, Composites Part A: Applied Science and Manufacturing, 82, 2016, pp. 159-169 (DOI: 10.1016/j.compositesa.2015.12.011).

[13] Cao, J., R. Akkerman, P. Boisse, J. Chen, H.S. Cheng, E.F. de Graaf, J.L. Gorczyca, P. Harrison, G. Hivet, J. Launay, W. Lee, L. Liu, S.V. Lomov, A. Long, E. de Luycker, F. Morestin, J. Padvoiskis, 
X.Q. Peng, J. Sherwood, T. Stoilova, X.M. Tao, I. Verpoest, A. Willems, J. Wiggers, T.X. Yu, and B. Zhu, Characterization of mechanical behavior of woven fabrics: Experimental methods and benchmark results, Composites Part A: Applied Science and Manufacturing, 39(6), 2008, pp. 1037-1053 (DOI: 10.1016/j.compositesa.2008.02.016).

[14] Harrison, P., F. Abdiwi, Z. Guo, P. Potluri, and W.R. Yu, Characterising the shear-tension coupling and wrinkling behaviour of woven engineering fabrics, Composites Part A: Applied Science and Manufacturing, 43(6), 2012, pp. 903-914 (DOI: 10.1016/j.compositesa.2012.01.024).

[15] Hosseinia, A., M.H. Kashani, F. Sassani, A.S. Milani, and F.K. Ko, Identifying the distinct shear wrinkling behavior of woven composite preforms under bias extension and picture frame tests, Composite Structures, 185, 2018, pp. 764-773 (DOI: 10.1016/j.compstruct.2017.11.033).

[16] Alsayednoor, J., F. Lennard, W.R. Yu, and P. Harrison, Influence of specimen pre-shear and wrinkling on the accuracy of uniaxial bias extension test results, Composites Part A: Applied Science and Manufacturing, 101, 2017, pp. 81-97 (DOI: 10.1016/j.compositesa.2017.06.006).

[17] Harrison, P., E. Taylor, and J. Alsayednoor, Improving the accuracy of the uniaxial bias extension test on engineering fabrics using a simple wrinkle mitigation technique, Composites Part A: Applied Science and Manufacturing, 108, 2018, pp. 53-61 (DOI: 10.1016/j.compositesa.2018.02.025).

[18] Launay, J., G. Hivet, A.V. Duong, and P. Boisse, Experimental analysis of the influence of tensions on in plane shear behaviour of woven composite reinforcements, Composites Science and Technology, 68(2), 2008, pp. 506-515 (DOI: 10.1016/j.compscitech.2007.06.021).

[19] Harrison, P., M.F. Alvarez, and D. Anderson, Towards comprehensive characterisation and modelling of the forming and wrinkling mechanics of engineering fabrics, International Journal of Solids and Structures, 2017, pp. (DOI: 10.1016/j.ijsolstr.2016.11.008).

[20] Peirce, F.T., The "Handle" of Cloth as a Measurable Quantity, Journal of the Textile Institute Transactions, 21(9), 1930, pp. T377-T416 (DOI: 10.1080/19447023008661529).

[21] British Standard. Textiles: test methods for nonwovens, in Part 7: Determination of bending length. BS EN ISO 9073-7; 1998.

[22] Liang, B., P. Chaudet, and P. Boisse, Curvature determination in the bending test of continuous fibre reinforcements, Strain, 53(1), 2017, pp. e12213 (DOI: 10.1111/str.12213).

[23] de Bilbao, E., D. Soulat, G. Hivet, and A. Gasser, Experimental Study of Bending Behaviour of Reinforcements, Experimental Mechanics, 50(3), 2009, pp. 333-351 (DOI: 10.1007/s11340009-9234-9).

[24] Potluri, P. and J. Atkinson, Automated manufacture of composites: handling, measurement of properties and lay-up simulations, Composites Part A: Applied Science and Manufacturing, 34(6), 2003, pp. 493-501 (DOI: 10.1016/s1359-835x(03)00056-3).

[25] Bouquerel, L., N. Moulin, S. Drapier, P. Boisse, and J.-M. Beraud, Modelling and simulating the forming of new dry automated lay-up reinforcements for primary structures, AIP Conference Proceedings, 1896(1), 2017, pp. 030008 (DOI: 10.1063/1.5007995).

[26] Creech, G. and A.K. Pickett, Meso-modelling of Non-Crimp Fabric composites for coupled drape and failure analysis, Journal of Materials Science, 41(20), 2006, pp. 6725-6736 (DOI: 10.1007/s10853-006-0213-6).

[27] Gatouillat, S., A. Bareggi, E. Vidal-Sallé, and P. Boisse, Meso modelling for composite preform shaping - Simulation of the loss of cohesion of the woven fibre network, Composites Part A: Applied Science and Manufacturing, 54, 2013, pp. 135-144 (DOI: 10.1016/j.compositesa.2013.07.010).

[28] Charmetant, A., J.G. Orliac, E. Vidal-Salle, and P. Boisse, Hyperelastic model for large deformation analyses of 3D interlock composite preforms, Composites Science and Technology, 72(12), 2012, pp. 1352-1360 (DOI: 10.1016/j.compscitech.2012.05.006).

[29] Khan, M.A., T. Mabrouki, E. Vidal-Sallé, and P. Boisse, Numerical and experimental analyses of woven composite reinforcement forming using a hypoelastic behaviour. Application to the 
double dome benchmark, Journal of Materials Processing Technology, 210(2), 2010, pp. 378388 (DOI: 10.1016/j.jmatprotec.2009.09.027).

[30] Yu, W.R., M. Zampaloni, F. Pourboghrat, K. Chung, and T.J. Kang, Analysis of flexible bending behavior of woven preform using non-orthogonal constitutive equation, Composites Part A: Applied Science and Manufacturing, 36(6), 2005, pp. 839-850 (DOI: 10.1016/j.compositesa.2004.10.026).

[31] Boisse, P., J. Colmars, N. Hamila, N. Naouar, and Q. Steer, Bending and wrinkling of composite fiber preforms and prepregs. A review and new developments in the draping simulations, Composites Part B: Engineering, 141, 2018, pp. 234-249 (DOI: 10.1016/j.compositesb.2017.12.061).

[32] Dangora, L.M., C.J. Mitchell, and J.A. Sherwood, Predictive model for the detection of out-ofplane defects formed during textile-composite manufacture, Composites Part A: Applied Science and Manufacturing, 78, 2015, pp. 102-112 (DOI: 10.1016/j.compositesa.2015.07.011).

[33] Harrison, P., Modelling the forming mechanics of engineering fabrics using a mutually constrained pantographic beam and membrane mesh, Composites Part A: Applied Science and Manufacturing, 81, 2016, pp. 145-157 (DOI: 10.1016/j.compositesa.2015.11.005).

[34] Hamila, N., P. Boisse, F. Sabourin, and M. Brunet, A semi-discrete shell finite element for textile composite reinforcement forming simulation, International Journal for Numerical Methods in Engineering, 79(12), 2009, pp. 1443-1466 (DOI: 10.1002/nme.2625).

[35] Dörr, D., F.J. Schirmaier, F. Henning, and L. Kärger, A viscoelastic approach for modeling bending behavior in finite element forming simulation of continuously fiber reinforced composites, Composites Part A: Applied Science and Manufacturing, 94, 2017, pp. 113-123 (DOI: 10.1016/j.compositesa.2016.11.027).

[36] Döbrich, O., T. Gereke, O. Diestel, S. Krzywinski, and C. Cherif, Decoupling the bending behavior and the membrane properties of finite shell elements for a correct description of the mechanical behavior of textiles with a laminate formulation, Journal of Industrial Textiles, 44(1), 2013, pp. 70-84 (DOI: 10.1177/1528083713477442).

[37] Budiansky, B., J.L. Sanders, H.U.D.o. Engineering, A. Physics, and U.S.O.o.N. Research, On the "best" First-order Linear Shell Theory. 1962: Division of Engineering and Applied Physics, Harvard University.

[38] CloudCompare 2.11. Retrieved from http://www.cloudcompare.org. 2016.

[39] Pourtier, J., B. Duchamp, M. Kowalski, P. Wang, X. Legrand, and D. Soulat, Two-way approach for deformation analysis of non-crimp fabrics in uniaxial bias extension tests based on pure and simple shear assumption, International Journal of Material Forming, 2019, pp. (DOI: 10.1007/s12289-019-01481-8). 


\section{Tables}

Table 1: Fabric parameters of the NCF (Hexcel FCIM359).

\begin{tabular}{lll}
\hline & Properties & \\
\hline Fibre type & Toray T620-50 C \\
Fabric areal mass $(\mathrm{gsm})$ & 440 \\
Fibre orientation $\left({ }^{\circ}\right)$ & \pm 45 \\
Tow size $(\mathrm{K})$ & 24 \\
Average tow width $(\mathrm{mm})$ & 1.50 \\
Stitch yarn & Polyester & Pillar \\
Stitch pattern & 0 & 0.4 \\
Stitch orientation $\left({ }^{\circ}\right)$ & &
\end{tabular}

Table 2: Material properties for model input.

\begin{tabular}{cclcc}
\hline Test & $\begin{array}{c}\text { Dimension } \\
(\mathrm{mm})\end{array}$ & Material & $\begin{array}{c}\text { Modulus } \\
(\mathrm{GPa})\end{array}$ & Bending stiffness (Nm) \\
\hline Bias extension & $200 \times 400$ & Woven [19] & $3.0[19]$ & $0.0002[19]$ \\
Picture frame shear & $\begin{array}{c}\text { Frame: } 145 \times 145 \\
\text { Shear region:110 } \times 110\end{array}$ & NCF [6] & 3.0 & see Section 2.2 \\
\hline
\end{tabular}




\section{Figures}

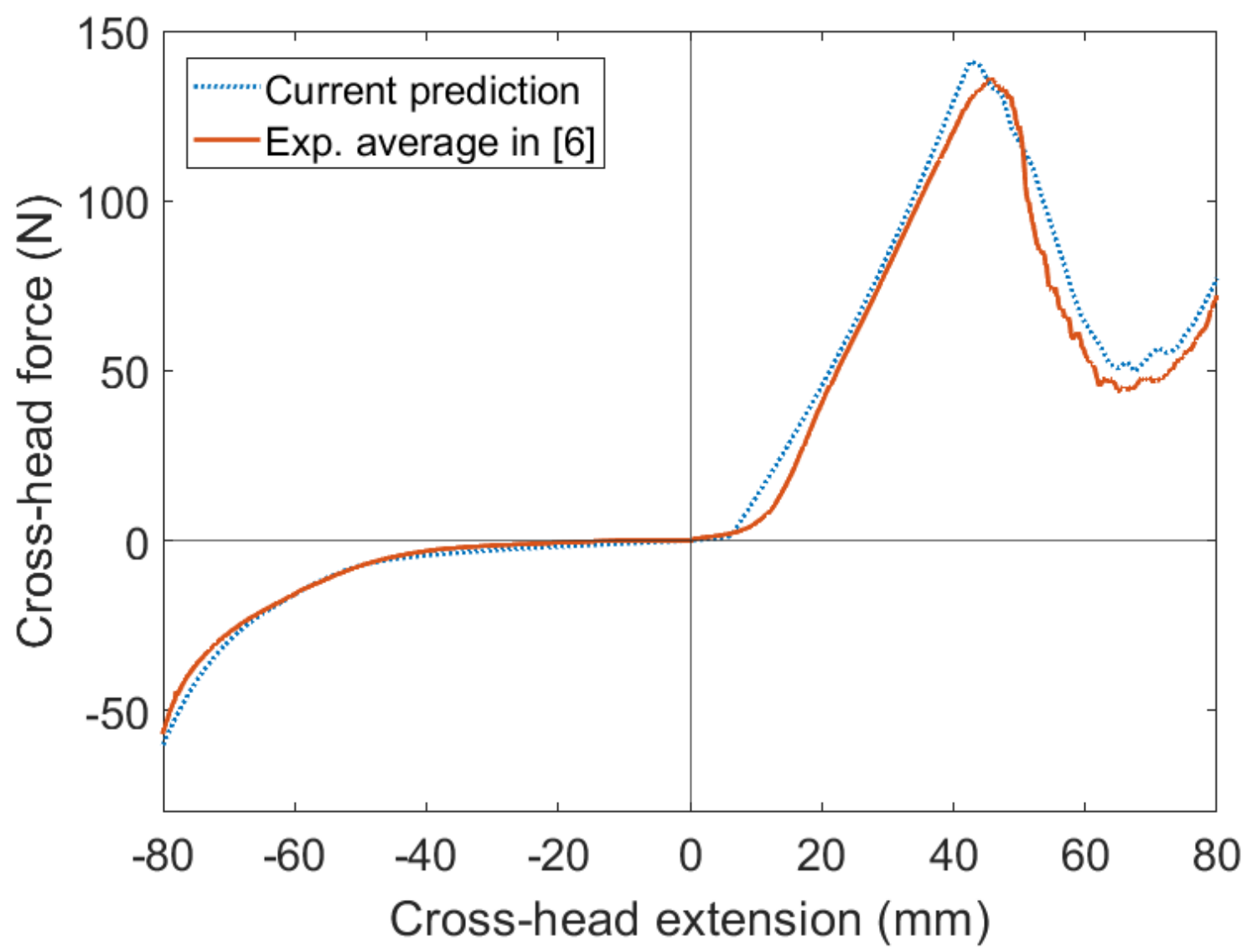

Figure 1: Comparison of experimental and predicted in-plane shear compliance curves for FCIM359 NCF.

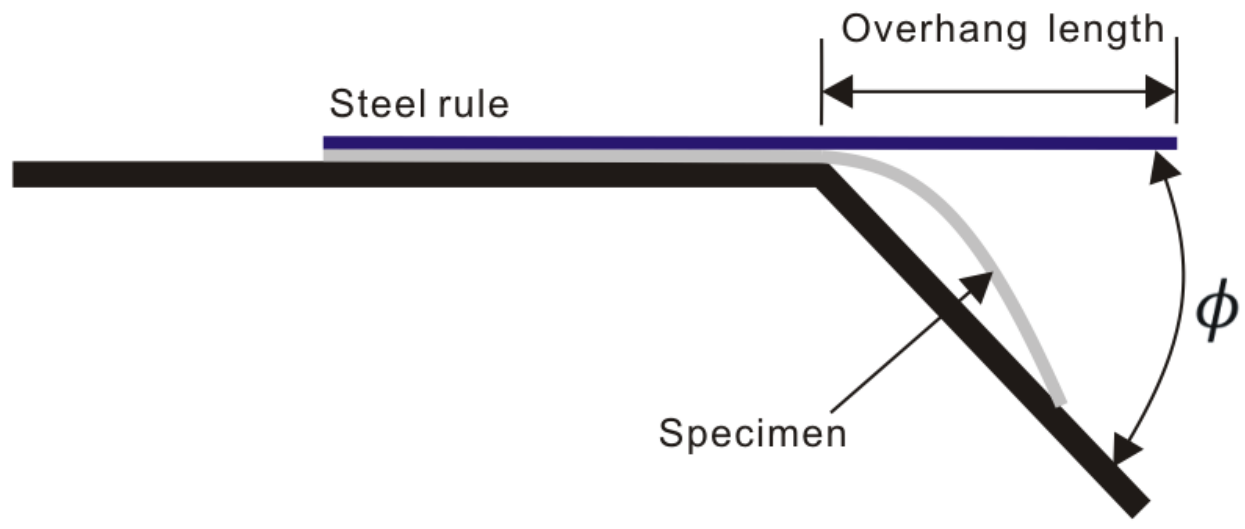

Figure 2: Schematic of the standard cantilever test in [21]. 


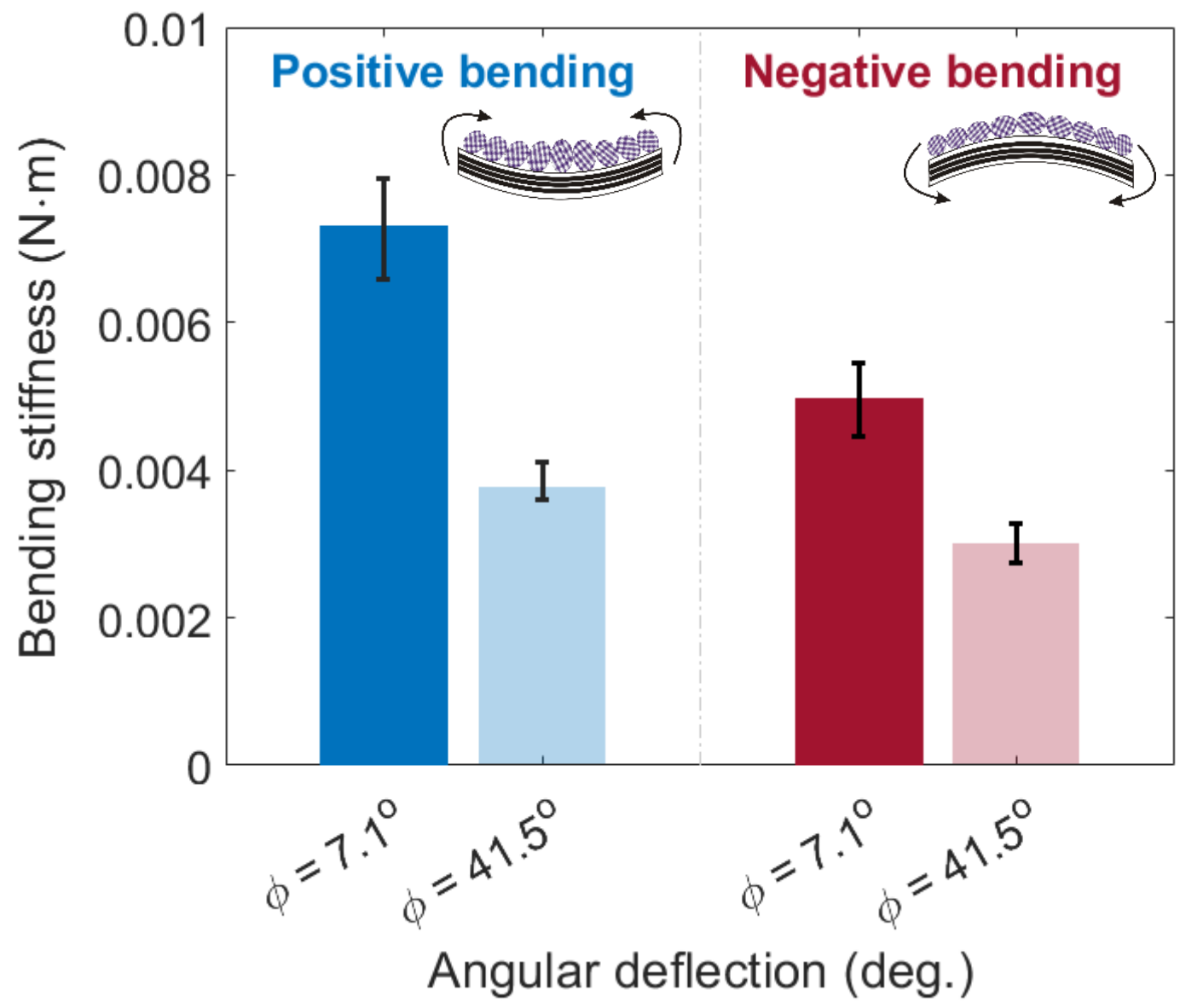

Figure 3: Constant bending rigidities measured for positive (blue) and negative (red) bending configurations at angular deflections of $7.1^{\circ}$ and $41.5^{\circ}$. 


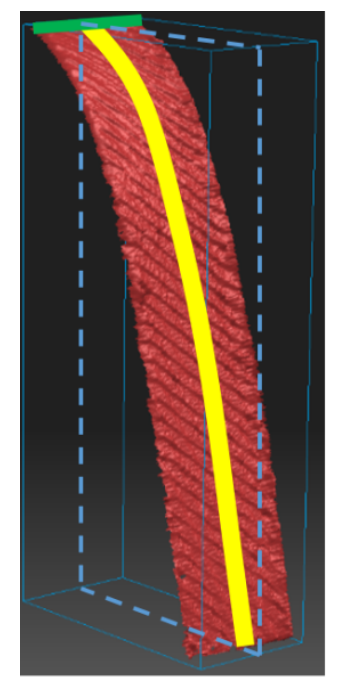

(a)

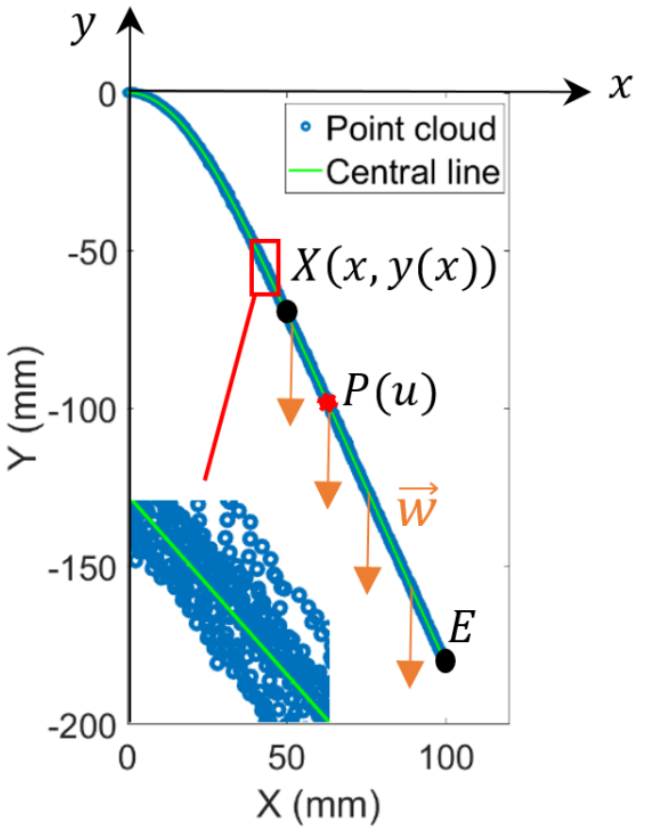

(b)

Figure 4: Bending stiffness characterisation using structured light scanning: (a) point cloud from scanning and (b) central profile fitting.

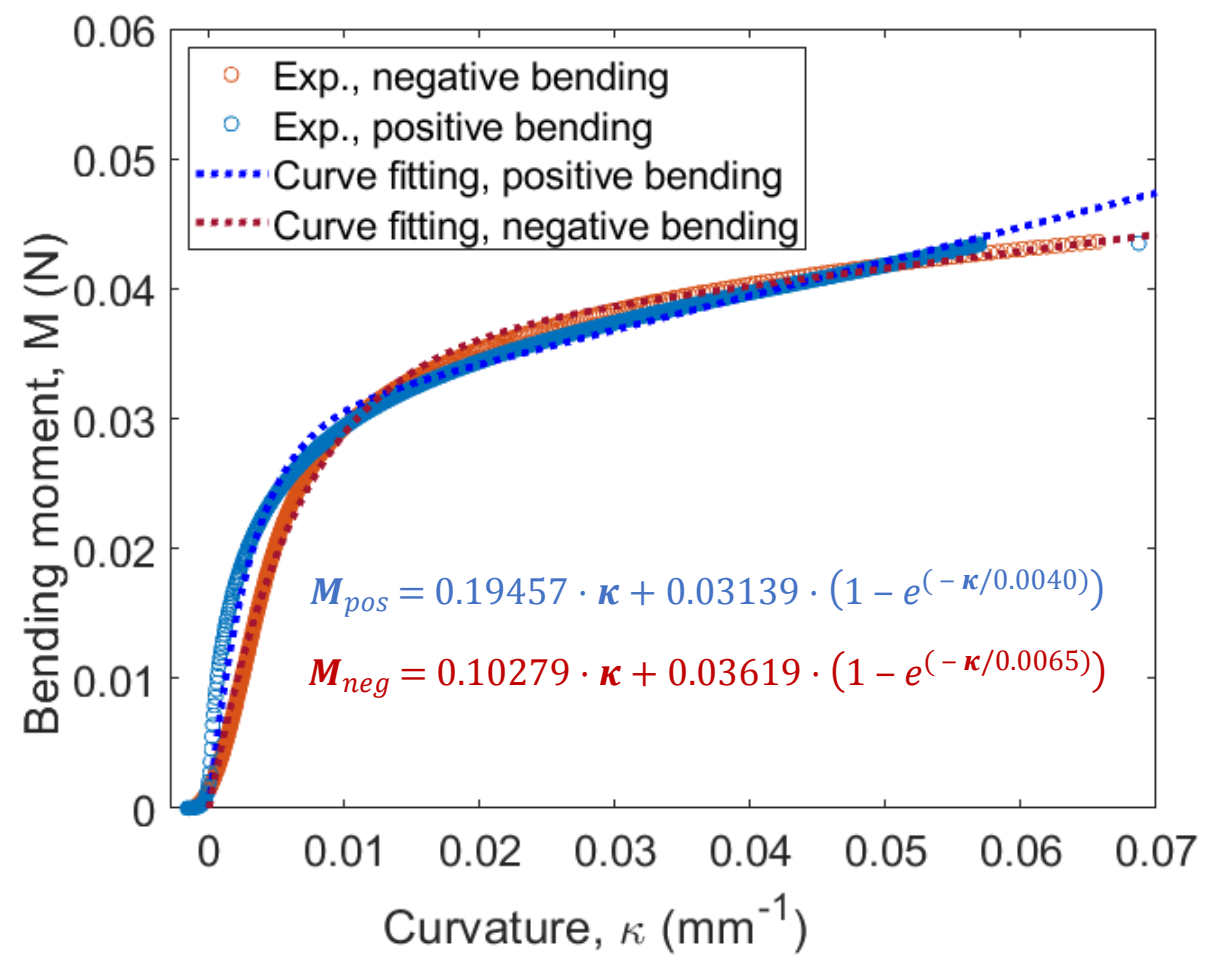

Figure 5: Bending moment vs. curvature curve characterised by revised cantilever test for the NCF (FCIM359) under positive (blue) and negative (red) bending. Expressions for both bending configurations were obtained by curve fitting using Voce's model [25]. 


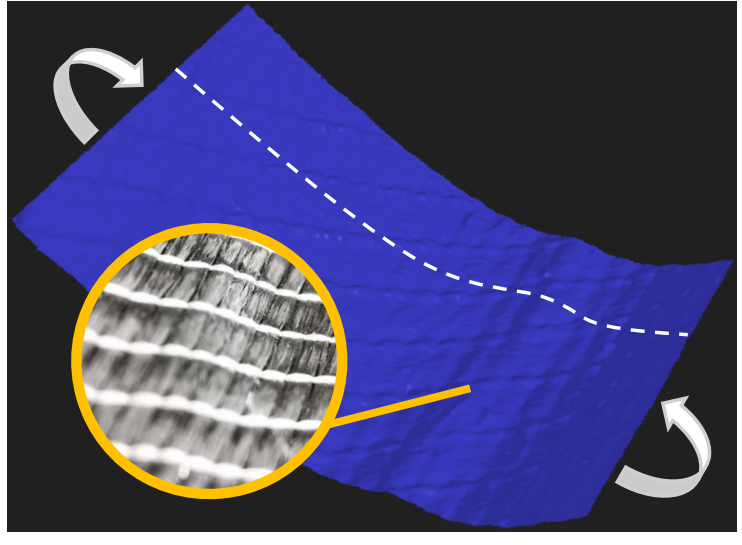

(a)

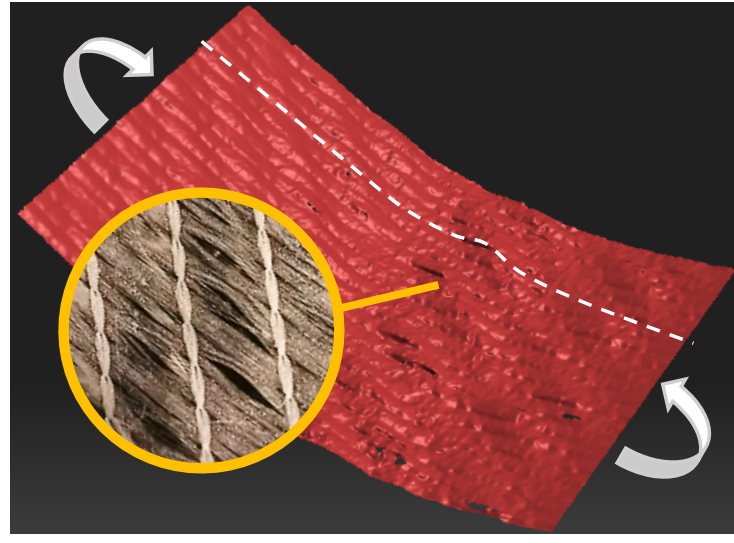

(b)

Figure 6: Surface scans of the compressive side of cantilevered bend specimens under (a) positive and (b) negative bending. Inset photos show details of fibre buckling.
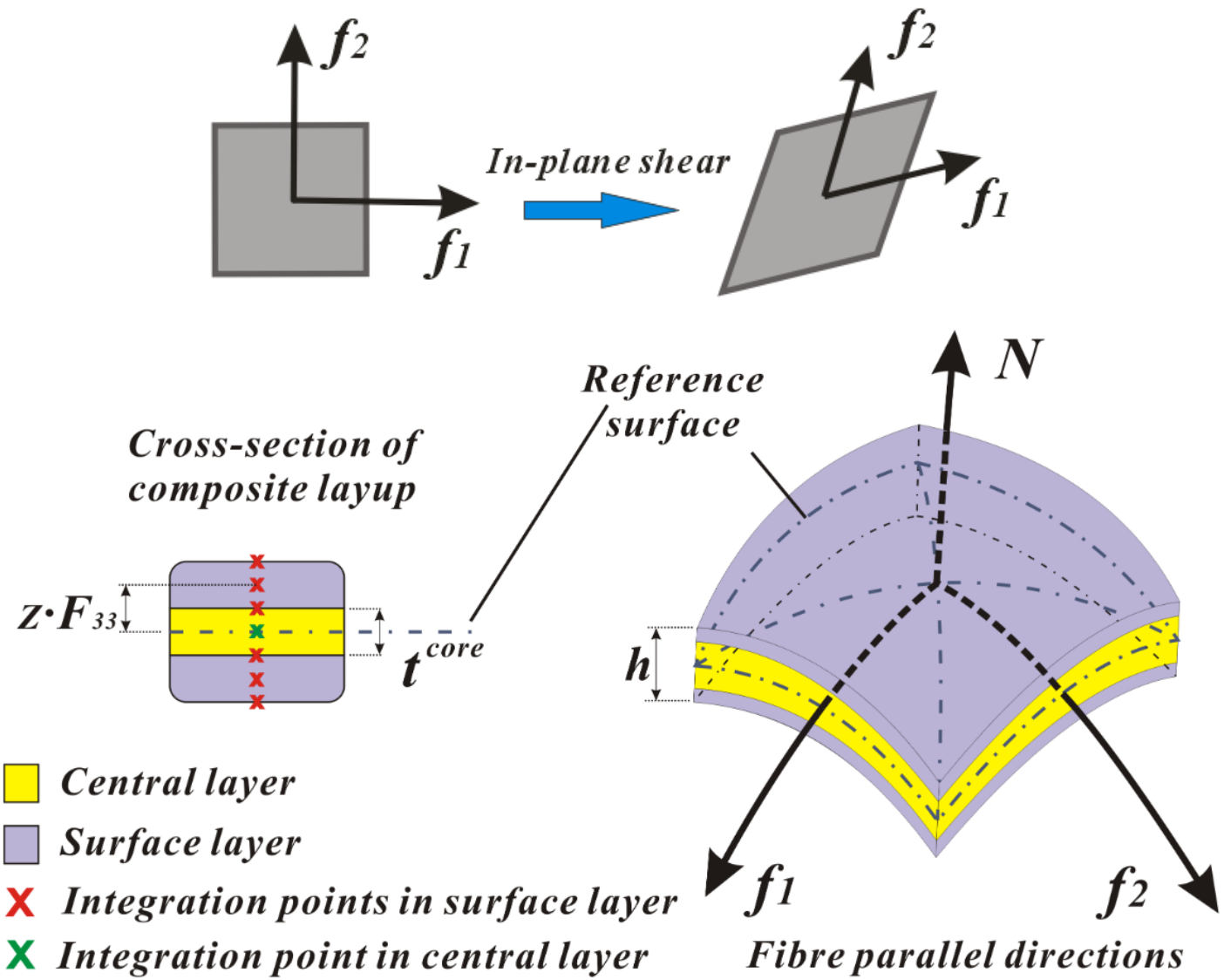

Figure 7: Schematic diagram of the shear deformation of biaxial fabric unit cell and the laminate layup model for membrane/bending stiffness decoupling. 


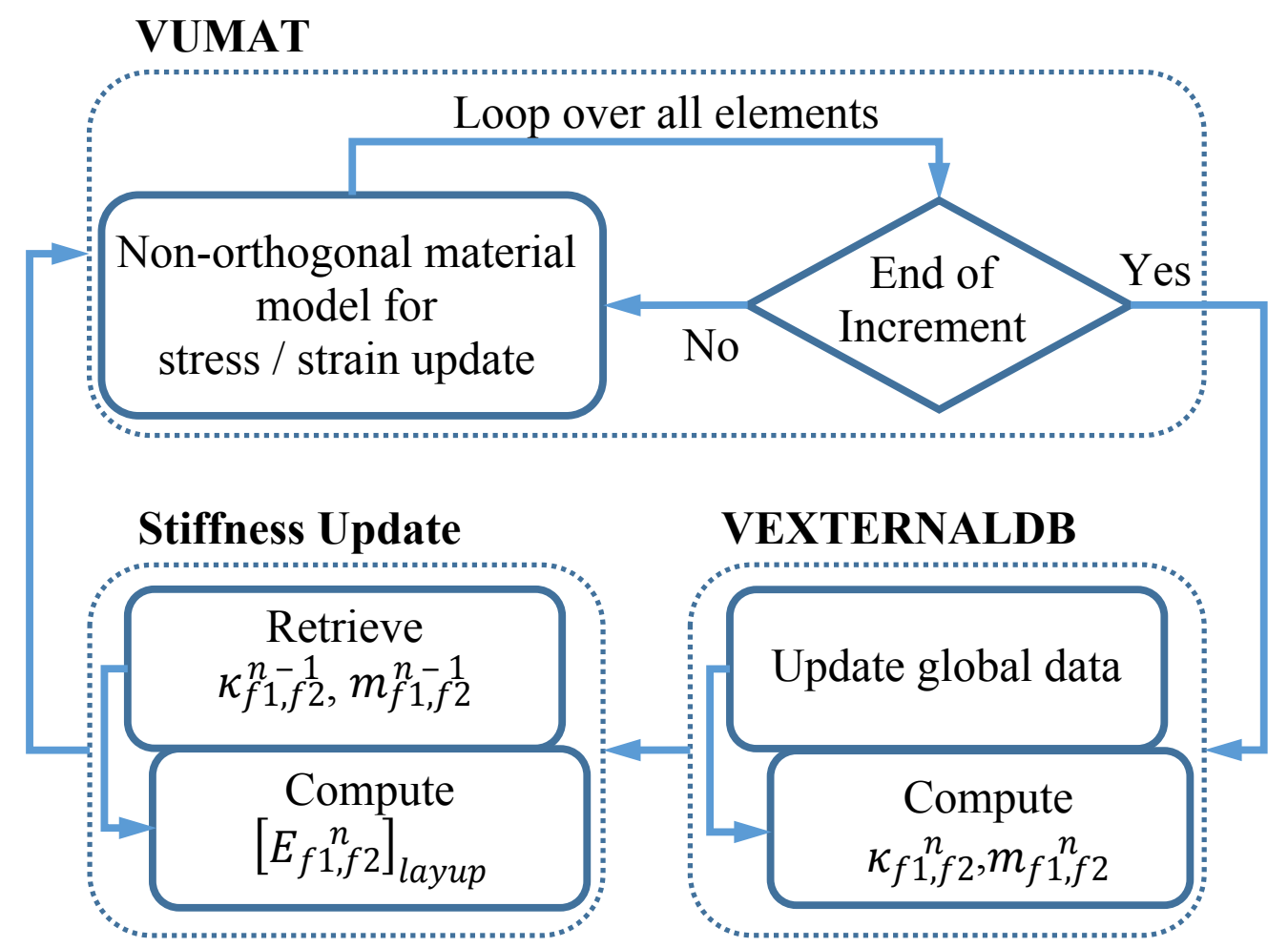

Figure 8: Flowchart for the implementation of the non-linear bending stiffness into Abaqus VUMAT: bending moment and curvature update. 


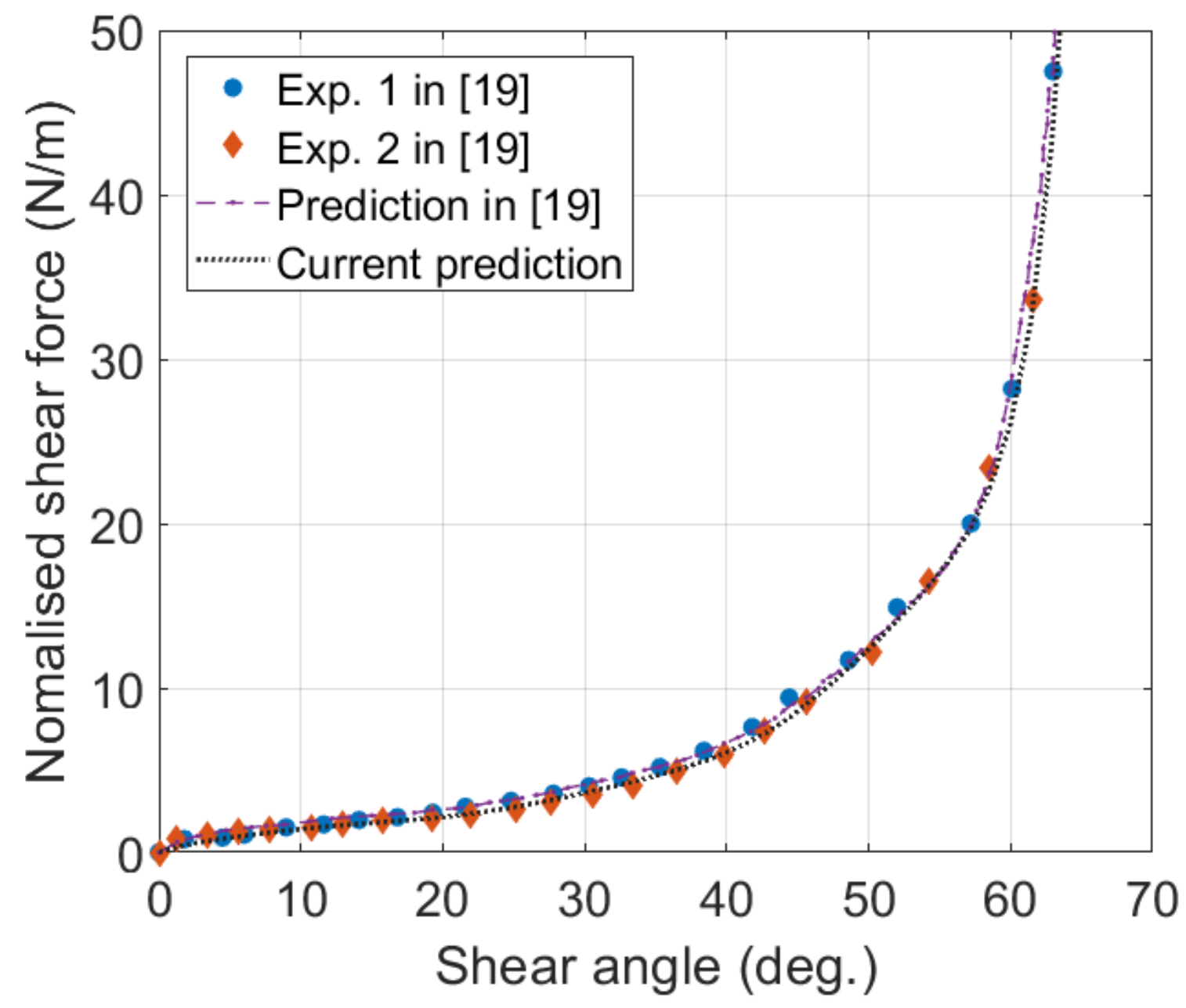

Figure 9: Normalised in-plane shear force vs. shear angle curve predicted by the current model. Experimental data and alternative simulation data taken from [19] for a woven fabric: CF-22-200-15. 


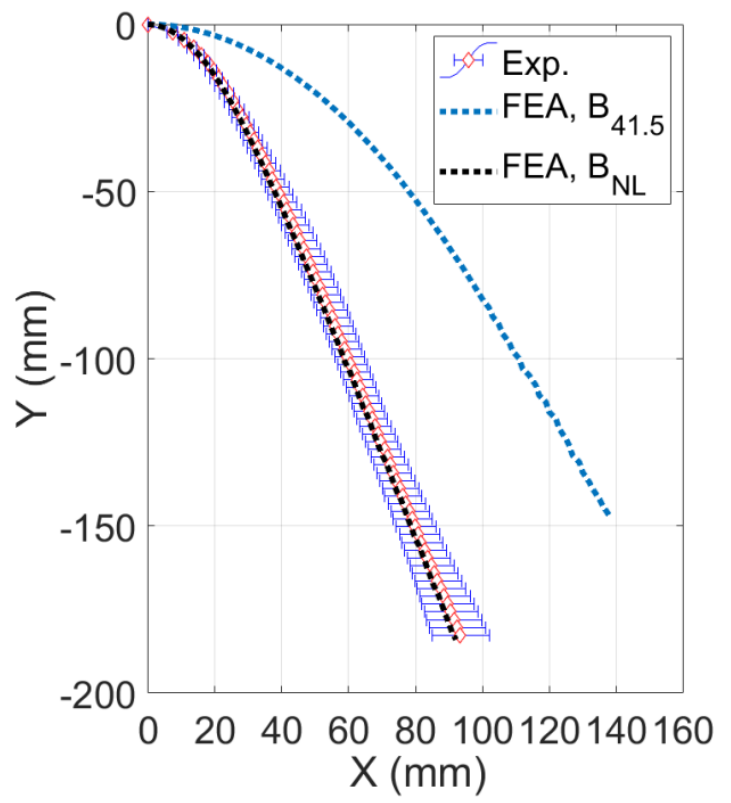

(a)

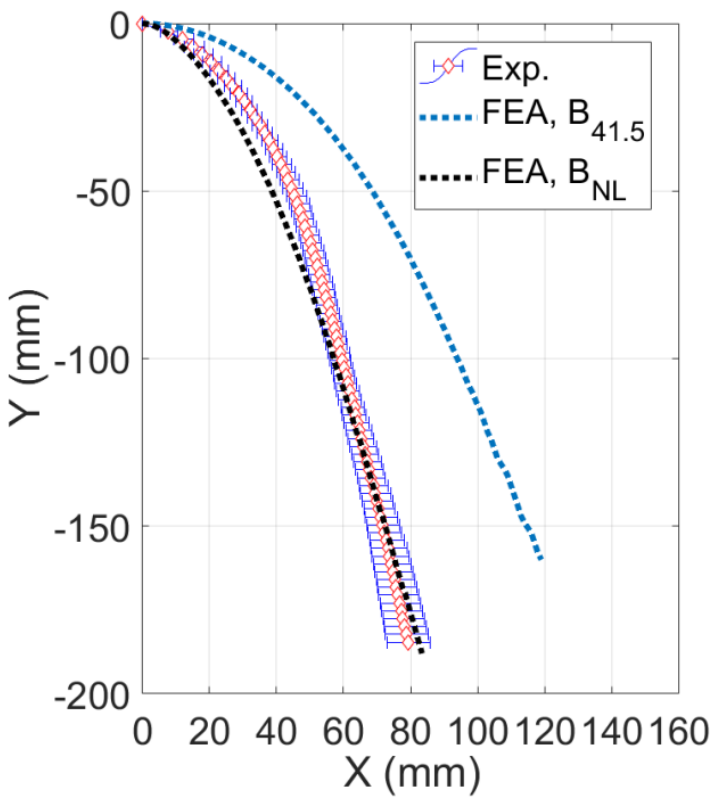

(b)

Figure 10: Comparison of bending deflections for experimental SWLS data (including error bars) and FEA predictions for (a) positive and (b) negative bending configurations. FEA predictions assume linear $\left(\mathrm{FEA}, \mathrm{B}_{41.5}\right)$ and non-linear (FEA, $\left.\mathrm{B}_{\mathrm{NL}}\right)$ bending stiffnesses. 
FE Simulation

Wrinkle amplitude

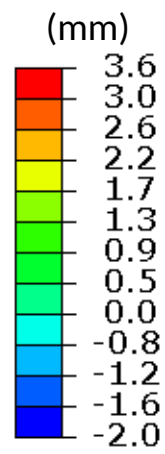

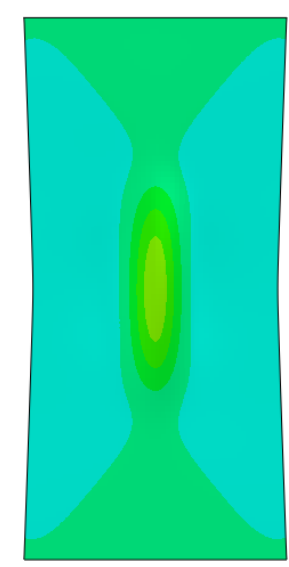

(Extension $=7.5 \mathrm{~mm})$

Wrinkle amplitude (mm)

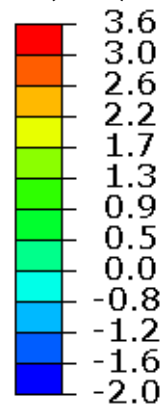

Wrinkle amplitude

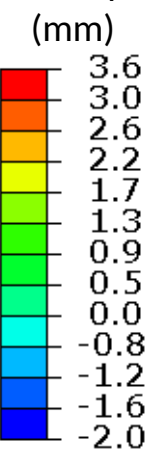

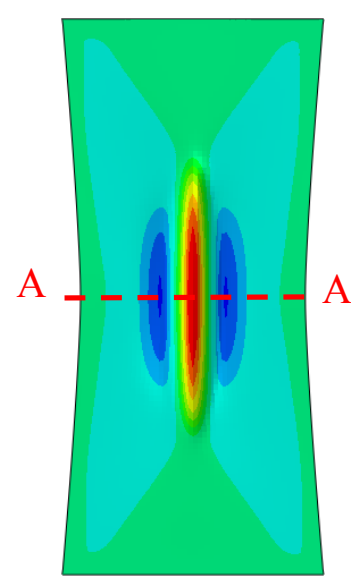

$($ Extension $=14.1 \mathrm{~mm})$

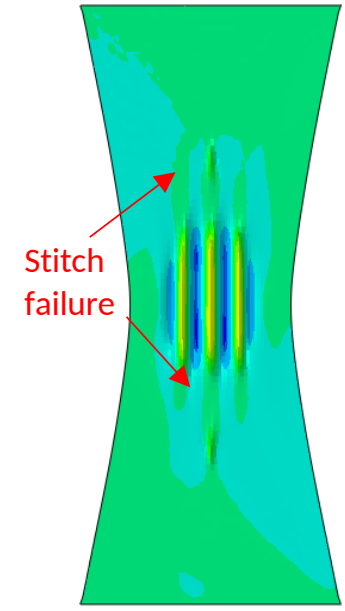

$($ Extension $=30.0 \mathrm{~mm})$

DIC measurement

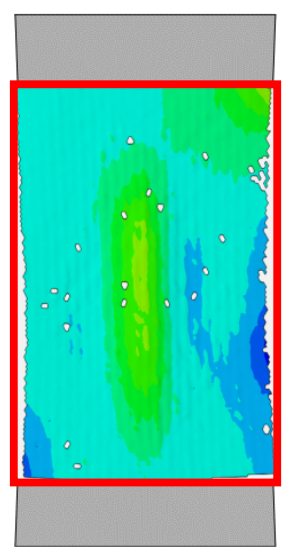

(Extension $=7.5 \mathrm{~mm})$

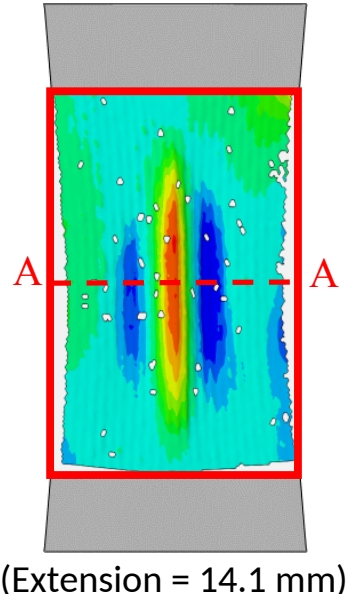

$($ Extension $=14.1 \mathrm{~mm})$
DIC tracking lost

$($ Extension $=30.0 \mathrm{~mm})$

Structured Light Scanning (SWLS)

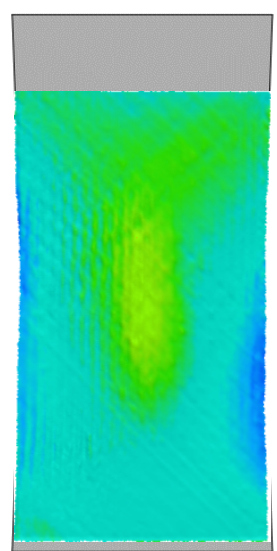

(Extension $=7.5 \mathrm{~mm})$

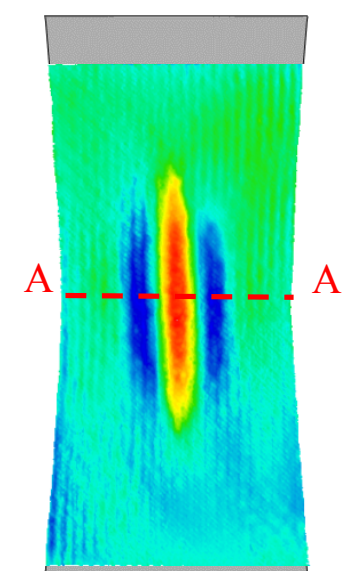

$($ Extension $=14.1 \mathrm{~mm})$

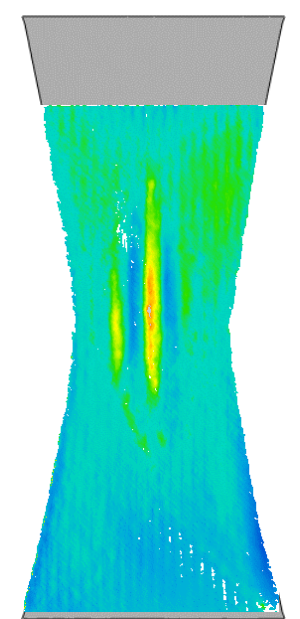

$($ Extension $=30.0 \mathrm{~mm})$

Figure 11: Out-of-plane wrinkling from bias-extension simulation (top row) against DIC (middle row) and SWLS (bottom row) measurements. 


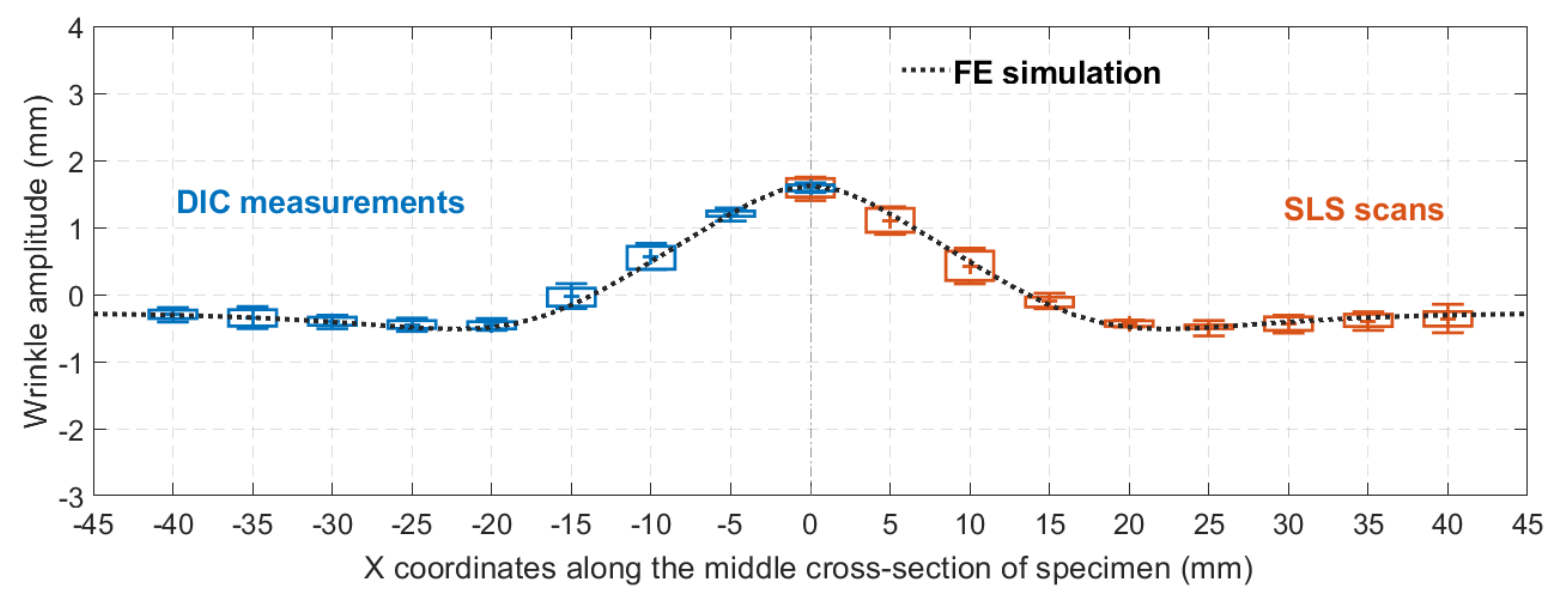

(a) Applied displacement $=7.5 \mathrm{~mm}$

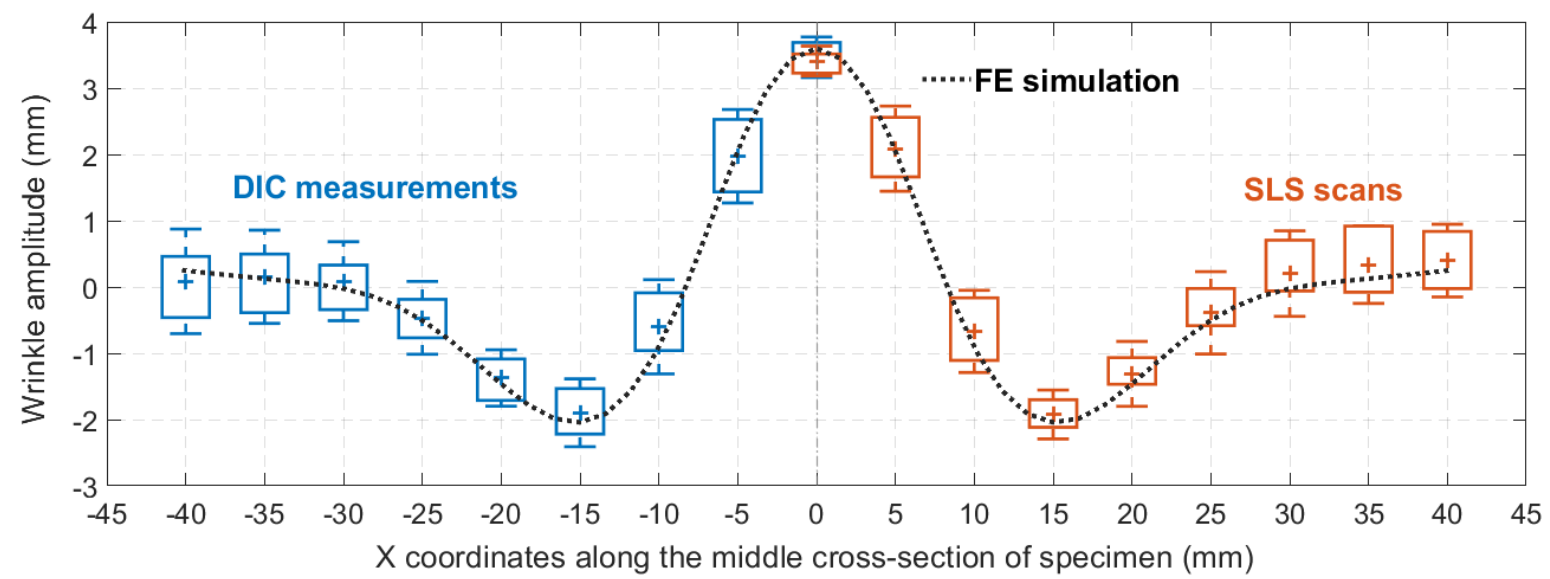

(b) Applied displacement $=14.1 \mathrm{~mm}$

Figure 12: Deformed cross-sectional shape (Section A-A in Figure 11) at the centre of a bias extension specimen. The dotted line represents the prediction from the FE simulation and the box plots represent data points measured by DIC and SWLS. 

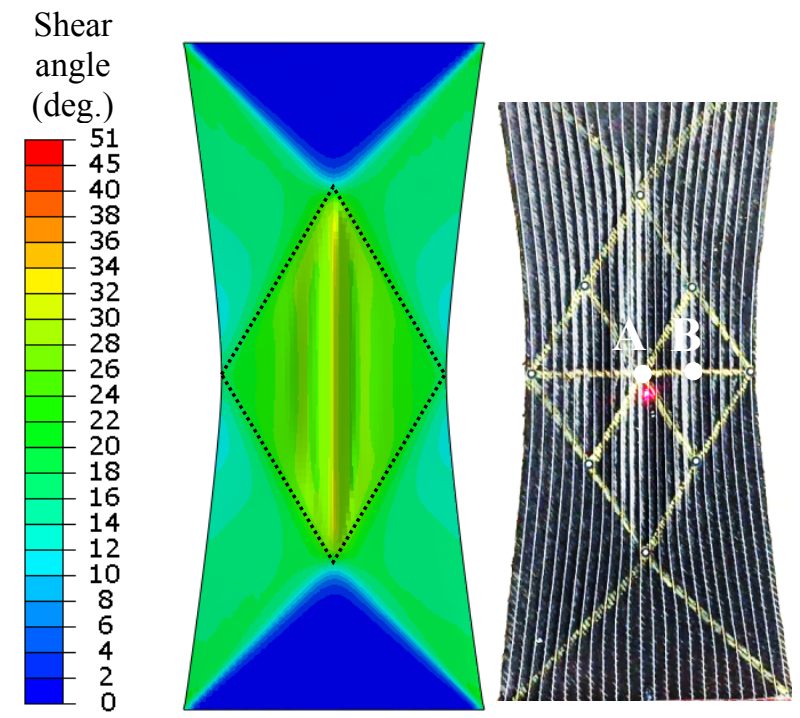

(a)
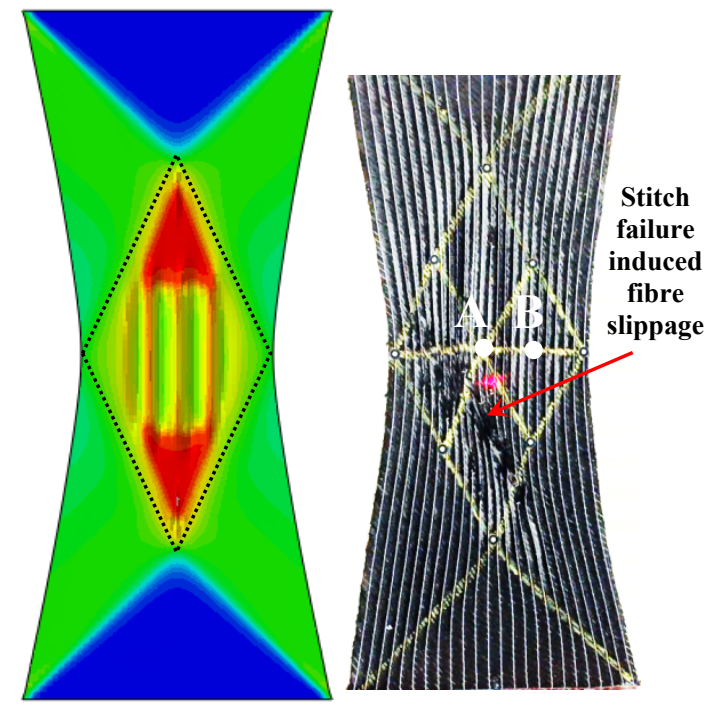
induced fibre slippage

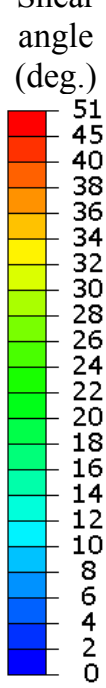

angle

(deg.)
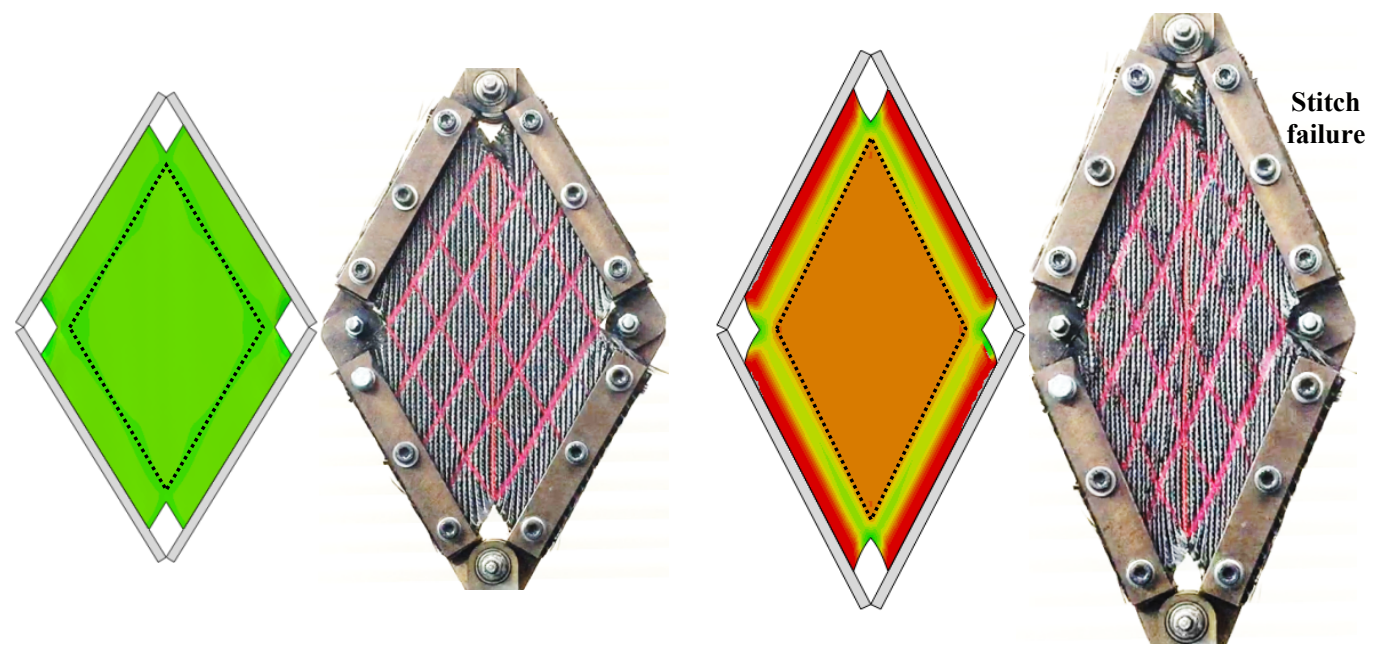

(b)

Figure 13: Comparison of predicted and measured shear deformation in (a) bias extension test and

(b) picture frame shear test. The right hand pair of images in each case indicate the shear

deformation following stitch rupture. The central diamond regions bounded by the black dotted lines indicate areas of interest for in-plane shear characterisation. 


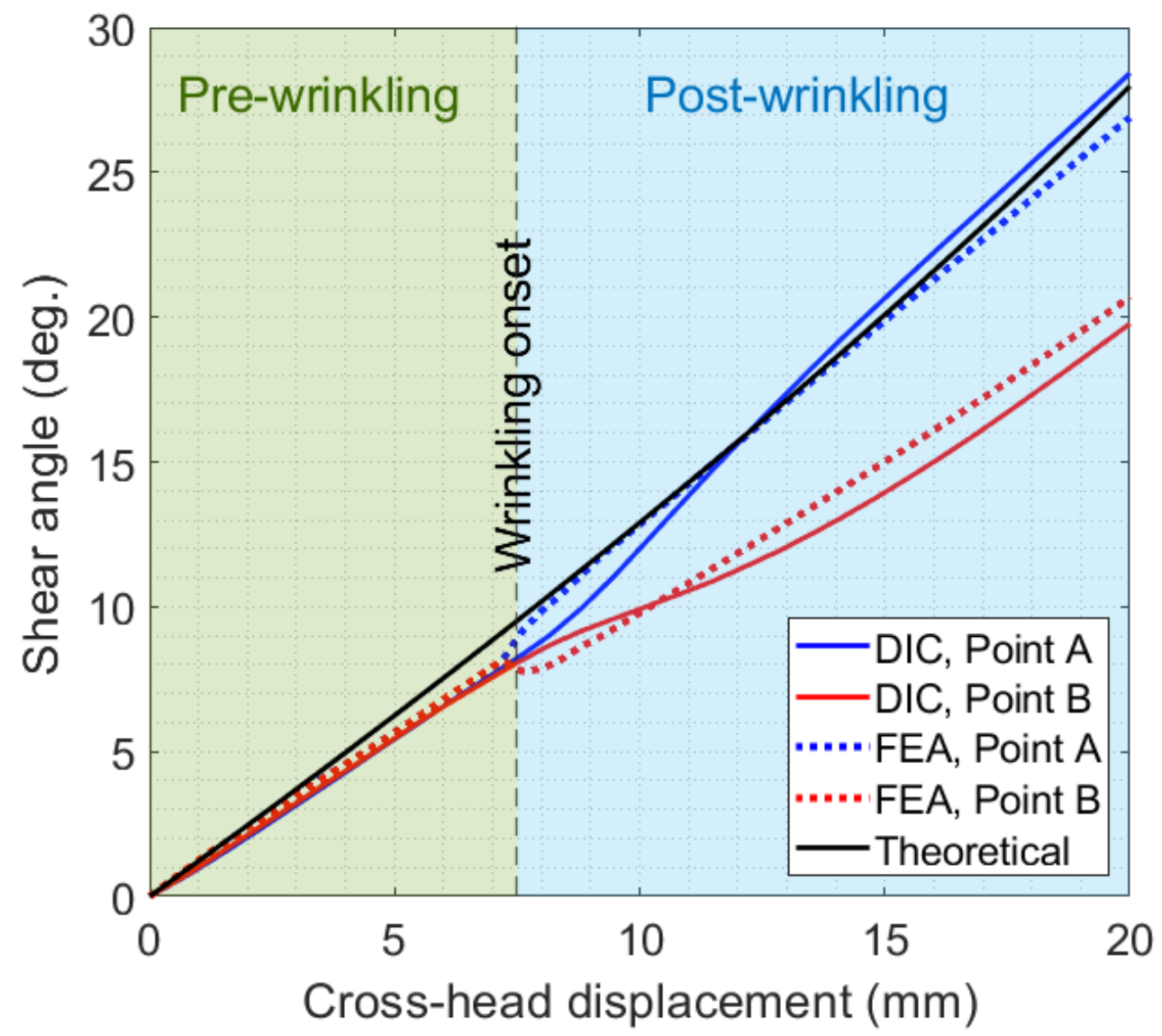

Figure 14: Shear angle vs. crosshead displacement curves derived from DIC, FEA and theoretical shear kinematics for Points A and B on the specimen in Figure 13. 


\section{Declaration of interests}

$X$ The authors declare that they have no known competing financial interests or personal relationships that could have appeared to influence the work reported in this paper. 
F. Yu - Conceptualisation, methodology, software, validation, investigation

S. Chen - Writing - Original draft, software

J.V. Viisainen - validation, investigation

M.P.F. Sutcliffe - Supervision, writing - review and editing

L.T. Harper - Supervision, writing - review and editing

N.A. Warrior - Supervision, Funding acquisition, writing - review and editing 